\title{
In Response to: Platelet-Rich Plasma Therapy and Antithrombotic Drugs
}

\section{Dear Editor:}

In our recent paper (1) we suggest that antithrombotic therapy should be suspended prior to undergoing PRP treatment. We thank "Insert authors" for bringing up an important counterpoint by citing two publications by Anitua et al.

The first article by Anitua et al (2) showed no difference in growth factors and degranulation process of platelets in patients taking anti-inflammatory and anticoagulants. This study is limited by a very small sample size in a narrow patient population (maxillofacial surgery) and a lack of clinical outcomes.

The second article by Anitua et al (3) provides valuable information regarding patients taking a compound similar to warfarin (acenocoumarol) and acetyl salicylic acid. This study, which is also limited by a small number of participants and a lack of clinical outcomes, only showed a modest effect on platelet growth factors.

Additionally, both of the Anitua studies utilized a method for obtaining PRP that is not necessarily reflective of the approach typically employed in most clinical practice.

One of the most prominent issues in the rapidly growing field of PRP therapy is that there is no stan- dardization of preparation. However, most classifications systems (4-6) consider platelets to be an indispensible component of the injectate. This is due to the fact that the therapeutic potential of PRP is thought to be based on the premise that growth factors from the alpha granules of platelets in supraphysiologic amounts can augment the healing response $(7,8)$.

In addition, other elements related to platelets, such as platelet activation and concentration may impact the clinical utility of PRP (4) Therefore, until we reach a better understanding of the underlying mechanisms involved in PRP induced healing and analgesia, it may be prudent to avoid the inhibition of platelets, as we recommend. Clearly, we agree that in patients where anti-coagulation therapy can not be stopped, as in Dr. Di Matteo et al's study (9), proceeding with PRP is still an option.
Houman Danesh, MD
Mount Sinai Hospital
5 East 98th St 6th Floor
New York, NY 10029
E-mail: houmanmd@gmail.com

\section{References}

1. Ramsook RR, Danesh H. Timing of Platelet Rich Plasma Injections During Antithrombotic Therapy. Pain Physician 2016:19: E1055-1061.

2. Anitua E, Troya M, Zalduendo M, Orive $G$. The effect of different drugs on the preparation and biological outcomes of plasma rich in growth factors. Ann Anat. 2014; 196: 423-429

3. Anitua E, Troya M, Zalduendo M, Orive G. Effects of anti-aggregant, anti-inflammatory and anti-coagulant drug consumption on the preparation and therapeutic potential of plasma rich in growth factors (PRGF). Growth Factors 2015:33: 57-64.
Mautner K, Malanga G, Smith J, Shiple B, Ibrahim V, Sampson S, Bowen J. A call for a standard Classification system for future biologic research: the rationale for new PRP nomenclature. PMR 2015; 7:S53-S59.

5. Megalon J, Chateau A, Bertrand B, Louis, M, Silvestre A, Giraudo L, Veran J, Sabatier F. DEPA classification: a proposal for standardising PRP use and a retrospective application of available devices. BM] Open Sport Exerc Med 2016; 2.

6. Masayuki Y, Natsumi W, Kazuo Y. PRP therapy classification (Letter to the Editor). Regenerative Therapy 2015; 2(1).
7. Alsousou J, Thompson M, Hulley P, Noble A, Willett K. The Biology of plateletrich plasma and its application in trauma and orthopaedic surgery: a review of the literature. J Bone Joint Surg Br 2009; 91:987-96.

8. Mishra A, Woodall J Jr, Vieira A. Treatment of Tendon and muscle using platelet-rich plasma. Cin Sports Med 2009; 28:113-125.

9. Di Matteo B, Filardo G, Lo Presti M, Kon E, Marcacci M. Chronic anti-platelet therapy: a contraindication for platelet-rich plasma intra-articular injections? Eur Rev Med Phamracol Sci 2014; 18:55-59. 